A Meta-Analysis of Conditioned Fear Generalization in Anxiety-Related Disorders Samuel E. Cooper, $\mathrm{PhD}^{1}$, Eva A. M. van Dis, $\mathrm{MSc}^{2}$, Muriel A. Hagenaars, $\mathrm{PhD}^{2}$, AngelosMiltiad is Krypotos, $\mathrm{PhD}^{2}$, Charles B. Nemeroff, $\mathrm{MD}, \mathrm{PhD}^{1,3,4}$, Shmuel Lissek, $\mathrm{PhD}^{5}$, Iris M. Engelhard, $\mathrm{PhD}^{2}$, \& Joseph E. Dunsmoor, $\mathrm{PhD}^{1,6}$

${ }^{1}$ Department of Psychiatry and Behavioral Sciences, University of Texas at Austin Austin, TX, USA

${ }^{2}$ Department of Clinical Psychology, Utrecht University, Utrecht, the Netherlands

${ }^{3}$ Institute of Early Life Adversity Research, University of Texas at Austin, Austin, TX, USA

${ }^{4}$ Mulva Clinic for the Neurosciences, University of Texas at Austin, Austin, TX, USA

${ }^{5}$ Department of Psychology, University of Minnesota - Twin Cities, Minneapolis, MN, USA

${ }^{6}$ Institute for Neuroscience, University of Texas at Austin, Austin, TX, USA

Contact: samuel.cooper@austin.utexas.edu; joseph.dunsmoor@austin.utexas.edu

Draft Version 2.0 submitted for review. This paper has not been peer-reviewed. Please do not copy or cite without the authors' permission. Data and supplemental materials are available at https://osf.io/89gjr/ 


\begin{abstract}
Objective: Generalization of conditioned fear is adaptive in some situations but maladaptive when fear excessively generalizes to innocuous stimuli with incidental resemblance to a genuine threat cue. Recently, empirical interest in fear generalization as a transdiagnostic explanatory mechanism underlying anxiety-related disorders has accelerated. As there are now several studies of fear generalization across multiple types of anxiety-related disorders, the authors conducted a metaanalysis of studies reporting behavioral measures (subjective ratings and psychophysiological indices) of fear generalization in anxiety-related disorder vs. comparison groups.

Method: We conducted systematic searches of electronic databases (conducted from JanuaryOctober 2020) for fear generalization studies involving anxiety-related disorder groups or subclinical analogue groups. A total of 300 records were full-text screened and two unpublished datasets were obtained, yielding 16 studies reporting behavioral fear generalization. Randomeffects meta-analytic models and meta-regressions were applied to the behavioral data.

Results: Fear generalization was significantly heightened in anxiety-related disorder participants $(\mathrm{N}=439)$ relative to comparison participants $(\mathrm{N}=428)$. We did not identify any significant clinical, sample, or methodological moderators.

Conclusion: Heightened fear generalization is quantitatively supported as distinguishing anxietyrelated disord er groups from comparison groups. Evidence suggests this effect is transdiagnostic, relatively robust to experimental or sample parameters, and that generalization paradigms are a well-supported framework for neurobehavioral investigations of learning and emotion in anxietyrelated disorders. We discuss these findings in the context of prior meta-analyses and future directions and challenges for the field.
\end{abstract}


Animals, including humans, rarely reencounter the exact same threat, and therefore generalizing from prior threatening encounters is crucial for survival. For example, a soldier who encountered an explosion while in a military convoy might display increased readiness and high sensitivity for threat detection when in similar convoys in the future. However, overly broad threat generalization can hinder adaptive functioning when it promotes fear to stimuli or situations that are largely safe. For example, a veteran who witnessed a roadside bomb in a warzone might feel vigilant and severely anxious while stuck in traffic back home, symptoms characteristic of posttraumatic stress disorder (PTSD). Maladaptive generalization of learned fear is a core clinical feature in anxietyrelated disorders, which are among the most common and debilitating of psychopathologies (Bandelow \& Michaelis, 2015; Baxter et al., 2013). Although empirical demonstration of this clinically-relevant process dates back to the beginning of the previous century (Pavlov, 1927; J. B. Watson \& Rayner, 1920), by the mid-to-late twentieth-century, research efforts on stimulus generalization shifted almost entirely toward appetitive instrumental conditioning paradigms. A recent reemergence of empirical fear generalization research in laboratory animals and humans is due in large part to the continued relevance of conditioning-based models for the understanding and treatment of anxiety-related disorders (Pittig et al., 2018; Vervliet \& Boddez, 2020; Zinbarg et al., 2022), and initial research in clinical populations over the last decade provides preliminary evidence that overly broad behavioral (e.g., self-report ratings and psychophysiology) and neural generalization to an array of harmless stimuli is related to these psychopathologies (Dymond et al., 2015; Lissek, 2012). Yet, to date, the amount of empirical focus on fear generalization pales in comparison to research using other fear conditioning paradigms, such as extinction (Craske et al., 2018; Dunsmoor et al., 2015; Vervliet \& Boddez, 2020) 
Neuroscience investigations into fear generalization in healthy populations serve as a foundation for understand ing the emergence of increased generalization in clinical populations and provide avenues for potential treatment targets (Dymond et al., 2015; Lopresto et al., 2016; Pittig et al., 2018). One mechanistic account of fear generalization, for example, centers on the role of the hippocampus in pattern separation of similar but discrete experiences. Specifically, intense stress can compromise the hippocampus' ability to pattern separate, which in turn hinders discrimination of threat from safety and thus contributes to excessive generalization (Besnard \& Sahay, 2016). This indicates a possible target for intervention: addressing hippocampal dysfunction to limit fear generalization. A recent comprehensive model, inspired in part by LeDoux's 'low road' hypothesis of threat processing (LeDoux, 1994), centers on the role of subcortical (e.g., thalamic, amygdala, locus coeruleus) responses to stimuli that resemble a learned threat triggering pattern completion processes in the hippocampus (Webler et al., 2021). When coupled with impaired prefrontal inhibitory regulation, rapid threat detection along with pattem completion are proposed to lead to heightened generalization in anxiety-related disorders.

Importantly, neural models of pathological generalization are built almost entirely on preclinical animal models and knowledge of the symptomatology of various anxiety-related disorders — not on direct tests in anxiety-related disorder samples (Lissek, 2012). Put another way, current mechanistic models of fear generalization in anxiety-related disorders put the theoretical cart in front of the empirical horse. Fundamental assumptions that heightened fear generalization is a marker of anxiety-related disorders will benefit from systematic meta-analyses and reviews of empirical work linking fear generalization to neuropathophysiology. Such evidence could further propel the use of the fear generalization paradigm, as well as behavioral generalization as a viable treatment target, in a manner similar to the widespread use of fear extinction as both an 
experimental paradigm and explanatory construct for poor retention of corrective information in exposure-based therapy (Craske et al., 2018; Dunsmoor et al., 2015).

Central to efforts to map the etiology and pathophysiology of anxiety-related disorders are laboratory tasks that probe pathogenic mechanisms of fear and anxiety behavior and biology (Holmes et al., 2018). Differential fear conditioning remains the most common laboratory protocol to measure fear-based processes in humans. In the acquisition phase, one neutral stimulus (CS+) is paired with an aversive unconditioned stimulus (US), while another neutral stimulus is never paired with the US (CS-). Whereas extinction training represents the CS+ in the absence of the US, fear generalization tests introduce generalization stimuli (GS), which typically include several stimuli that vary in similarity to the CS+ and are never paired with the US (see Figure 1 for schematic). A practical benefit to generalization tests includes the ability to rapidly assess the effects of acquisition on subsequent learning and behavior. In contrast, deficits in extinction training between clinical and healthy populations are frequently identified only after a delay (between-session) during an extinction-recall test (Milad \& Quirk, 2012), although this can depend on the specific disorder (Cooper \& Dunsmoor, 2021; Lissek \& van Meurs, 2014; Zuj et al., 2016). Generalization tests are also likely better approximations of the clinical reality of anxiety pathology than simple differential paradigms (Beckers et al., 2013; Lissek et al., 2006). Those with anxiety-related disorders rarely, if ever, encounter the exact same threatening stimulus that previously frightened or traumatized them, nor one that always guarantees safety - the experience of living with pathological anxiety is much more uncertain and complex (Grupe \& Nitschke, 2013). Fear generalization offers a compelling framework for going beyond basic differential conditioning to understand pathological maladaptation in anxiety-related disorders. Given there are a growing number of studies of fear generalization in anxiety populations and conditioning 
work remains a source of innovation for advances in exposure therapies for clinical anxiety (Arch \& Abramowitz, 2015; Craske et al., 2018; Foa \& McLean, 2016), a meta-analysis of this work is warranted.

Figure 1. Schematic of differential fear acquisition, extinction, and generalization tests and response patterns related to anxiety-related disorders.

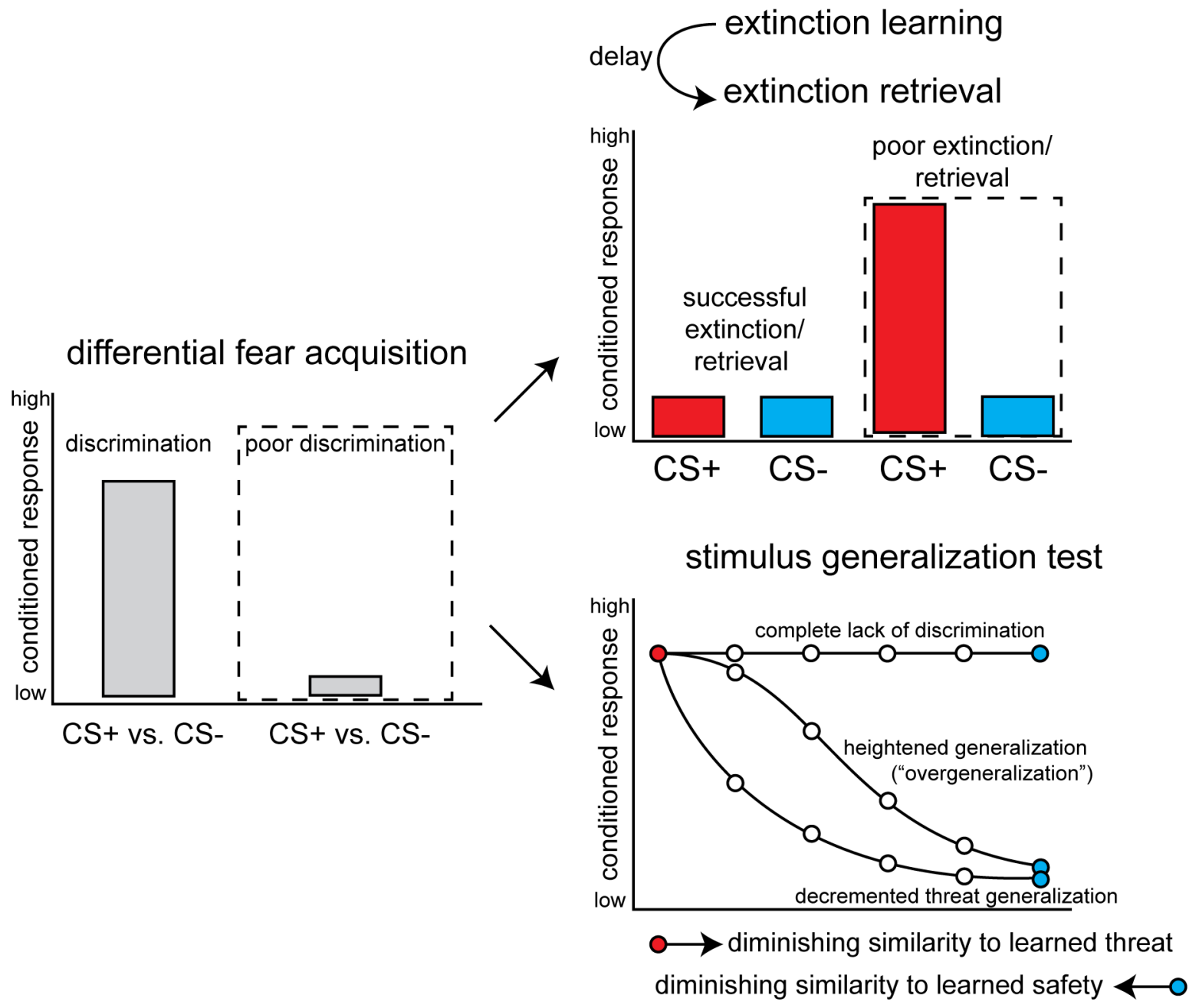

Notes. Figure includes hypotheticaldata that illustrates common differentialconditioning procedures. The left-most plot represents data from a basic differential fear acquisition design, with the left bardemonstrating successful discrimination between CS+ and CS-, and the right bardemonstrating poorer discrimination, as has been metaanalytically observed in anxiety-related disorders. The upper-right plot illustrates an extinction phase, either directly after acquisition or after a delay, in which the CS+ is no longer paired with the US. The left paired bars illustrate successful extinction, in which both CS+ and CS- responses are almost entirely diminished. The right paired bars illustrate poorer extinction, in which CS+ remains elevated, which has also been meta-analytically observed in anxiety-related disorders. The bottom-right plot illustrates a genera lization phase, which would replace the hypothetical extinction phase graphed above. In this genera lization phase, GSs that parametrically differ in similarity from the $\mathrm{CS}+$ are presented, and responding to these stimuli typically decreases in relation to the $\mathrm{CS}+$ response magnitude. Decremented genera lization (bottommost line), in which responding from the CS+ 
precipitously drops until nearCS- levels, is proposed to be characteristic of psychiatrically healthy/non-anxiety groups, whereas heightened genera lization (also referred to as overgenera lization, middle line) that involves relatively increased responding to GSs is linked to anxiety-related disorders. Monotonic responding (e.g., maximal response to all stimuli, topmost line) represents a failure to discriminate between stimuli and is not considered a

form of genera lization. $\mathrm{CS}+=$ conditioned threat cue; $\mathrm{CS}-=$ conditioned sa fety cue; GS = genera lization stimuli; US

$=$ unconditioned stimulus.

\section{The Current Meta-Analysis}

For fear generalization to transition from a specialty technique within the conditioning field to a widely recognized and used experimental paradigm for clinical research requires quantitative confirmation that heightened fear generalization is a consistent marker of anxiety psychopathology. Here, we present a meta-analysis of fear generalization in anxiety-related disorders. Prior meta-analytic reports of differential fear conditioning in anxiety disorders identified increased CS- responding during acquisition (Duits et al., 2015; Lissek et al., 2005), consistent, but not synonymous, with generalization to an unpaired cue while participants acquire fear. Prior reviews of fear generalization have not been quantitative in nature (Dunsmoor \& Paz, 2015; Dymond et al., 2015) or are of continuous anxiety-related traits and excluded pathological anxiety samples (Sep et al., 2019). Overall, the relation between heightened fear generalization and pathological anxiety remains unclear.

Our primary aim is to test group differences between anxiety-related disorder and comparison groups on behavioral and psychophysiological measures of fear generalization. We test standard group differences as a broad assessment of the overall effect size, which includes all identified studies. In a separate analysis, we test group differences in quadratic (i.e., curvilinear gradients of generalization that decrease from the CS+ to the CS-) effects, which are proposed as a more sensitive measure of heightened generalization (Lissek, 2012) but are not analyzed in all studies. We predict that anxiety-related disorder groups will demonstrate heightened fear generalization relative to comparison groups and that this effect will not be contingent on a 
particular disorder or experimental design parameter (Marin et al., 2020). We also evaluate differences in design and study quality and test their influence on generalization differences.

\section{Methods and Materials}

The current effort's methodology aligns with prior meta-analyses of fear conditioning and anxiety (Duits et al., 2015; Lissek et al., 2005; Sep et al., 2019). We preregistered in PROSPERO (CRD42021234718). Materials and data for this project are accessible at osf.io/89gjr.

\section{Search and Selection Strategy}

English-language records were selected through a systematic search of PubMed, PsycINFO, Embase, and OpenGrey (searches conducted January-October 2020) with a combination of terms related to conditioning (e.g., conditioning), fear (e.g., fear/Pavlovian/classical), and anxiety-related disorders (e.g., panic/phobi*). Table ST1 provides the complete search strategies. In addition, SC contacted several fear generalization experts to request data from potentially unpublished studies. Studies were included when they tested conditioned generalization among individuals with clinical or subclinical/subthreshold anxietyrelated disorders (i.e., PTSD, generalized anxiety disorder [GAD], panic disorder [PD], social anxiety disorder [SAD], specific phobia [SP], or obsessive-compulsive disorder [OCD]) relative to comparison groups without (subclinical/subthreshold levels of) anxiety-related disorders. Of note is that both PTSD and OCD have been removed from the Anxiety Disorder category for DSM5 (American Psychiatric Association, 2013). However, because conditioned fear generalization is clearly a component of PTSD and OCD (Cooper \& Dunsmoor, 2021; Zuj et al., 2016), they were included in our analysis and collectively referred to as anxiety-related disorders (Asmundson, 2019). See Supplementary Materials for full selection and screening details.

\section{Data Extraction and Quality Assessment (QA)}


Test statistics and $p$-values were extracted for each Group x Stimulus analysis for each dependent variable and for quadratic contrast interactions when available (56\% of identified studies). Clinical, demographic, and experimental details were extracted for all studies (see Supplementary Material for a list of extracted variables and coding information). Statistical data extraction was done by SC and independently verified by AMK. Study quality was measured using a custom standardized rubric (see Table ST2 for details) adapted fromprior work (Sep et al., 2019) and Cochrane Collaboration's risk of bias tool (Higgins et al., 2011; RoB 2, n.d.).

\section{Statistical Analyses}

All analyses were conducted with R (R Core Team, 2018). Hedge's $g$ was chosen as the group difference effect size given its robust properties across sample sizes and interpretability within meta-analytic frameworks (Lakens, 2013). Cluster-robust $p$ values and 95\% CIs are reported (Cameron \& Miller, 2015). Primary outcome measures were subjective (e.g., threat expectancy ratings, self-reported fear) and physiological measures of conditioned fear (e.g., fearpotentiated startle response [FPS] and skin conductance response [SCR]). Effect sizes were coded as positive in the case of increased generalization (determined through GS means relative to CS+ and CS- or, when not available, visual inspection using a standardized rubric that assesses the linearity of a gradient from CS+ to CS-, see Supplemental Material for full description) in the anxiety-related disorder group relative to comparisons. Other outcomes were coded as negative, yielding a conservative metric of anxiety vs. comparison differences that proportionally penalized any generalization result not clearly indicative of heightened generalization in anxiety groups relative to comparison participants.

See Supplemental Materials for full meta-analytic modeling details. Briefly, primary hypotheses were tested using three-level random-effects models (participant-level observations 
nested within effect size for each dependent variable, nested within study). One model included Group x Stimulus interaction effect sizes from all identified studies ${ }^{1}$ to provide; another included quadratic interaction effect sizes from the subset of studies that reported this test. Multiple effect sizes from the same study, corresponding to each dependent variable used to measure generalization, were nested within each study as a random effect. We tested stand ard heterogeneity statistics in both models. We then conducted moderation analyses using these models to statistically explain potential heterogeneity due to cross-study differences in clinical and experimental parameters (see list in Supplemental Materials). We tested for both omnibus moderation and for the significance of each level of the moderator (against zero). We also conducted meta-regressions that included a priori-defined continuous measures of clinical, methodological (including QA), and demographic fixed effects. False discovery rate correction was applied to all moderation and meta-regression tests per recommendations (Cafri et al., 2010). We tested for risk of publication bias using standard methods (see Supplemental Material).

\section{Results}

\section{Study Identification and Characteristics}

Figure $2 \mathrm{~A}$ depicts the flow of the selection and inclusion of studies. A total of 5,549 titles and abstracts were screened; 300 records were selected for full-text screening. An additional two unpublished studies were also obtained from fear generalization researchers (Cooper et al., unpub; Torrents et al., unpub). In total, sixteen studies reporting unique data met our inclusion criteria (total $N=867$, anxiety $n=439$, comparison $n=428, \mathrm{M}_{a g e}=30.1, \%$ women $=54 \%$, see Table 1 for full study details) (Ahrens et al., 2016; Greenberg et al., 2013; Kaczkurkin et al., 2017; Kaczkurkin \&

\footnotetext{
${ }^{1}$ For the $\mathrm{n}=3$ studies that did not report standard Group $\mathrm{x}$ Stimulus interactions for all dependent variables but did report quadratic analyses, we included these quadratic effects in the full data modeland later test if their effects significantly influenced results.
} 
Lissek, 2013; Lange, Goossens, Bakker, Michielse, Marcelis, et al., 2019; Lis et al., 2019; Lissek et al., 2010, 2014; Lissek \& Grillon, 2012; Morey et al., 2015, 2020; Neueder et al., 2019; Reichenberger et al., 2019; Tinoco-González et al., 2015). The following disorders were represented in the identified studies: $\operatorname{GAD}(n=82), \operatorname{OCD}(n=28), \operatorname{PD}(n=58), \operatorname{PTSD}(n=152), \operatorname{SAD}$ $(n=73)$, and SP $(n=46)$.

\section{QA Results}

Figure $2 \mathrm{~B}$ provides a summary graph of the overall risk of bias across published studies (unpublished studies were not evaluated, see Supplementary Materials for further description). 
Figure 2. PRISMA Flow Diagram and Overall Quality of Included Studies.

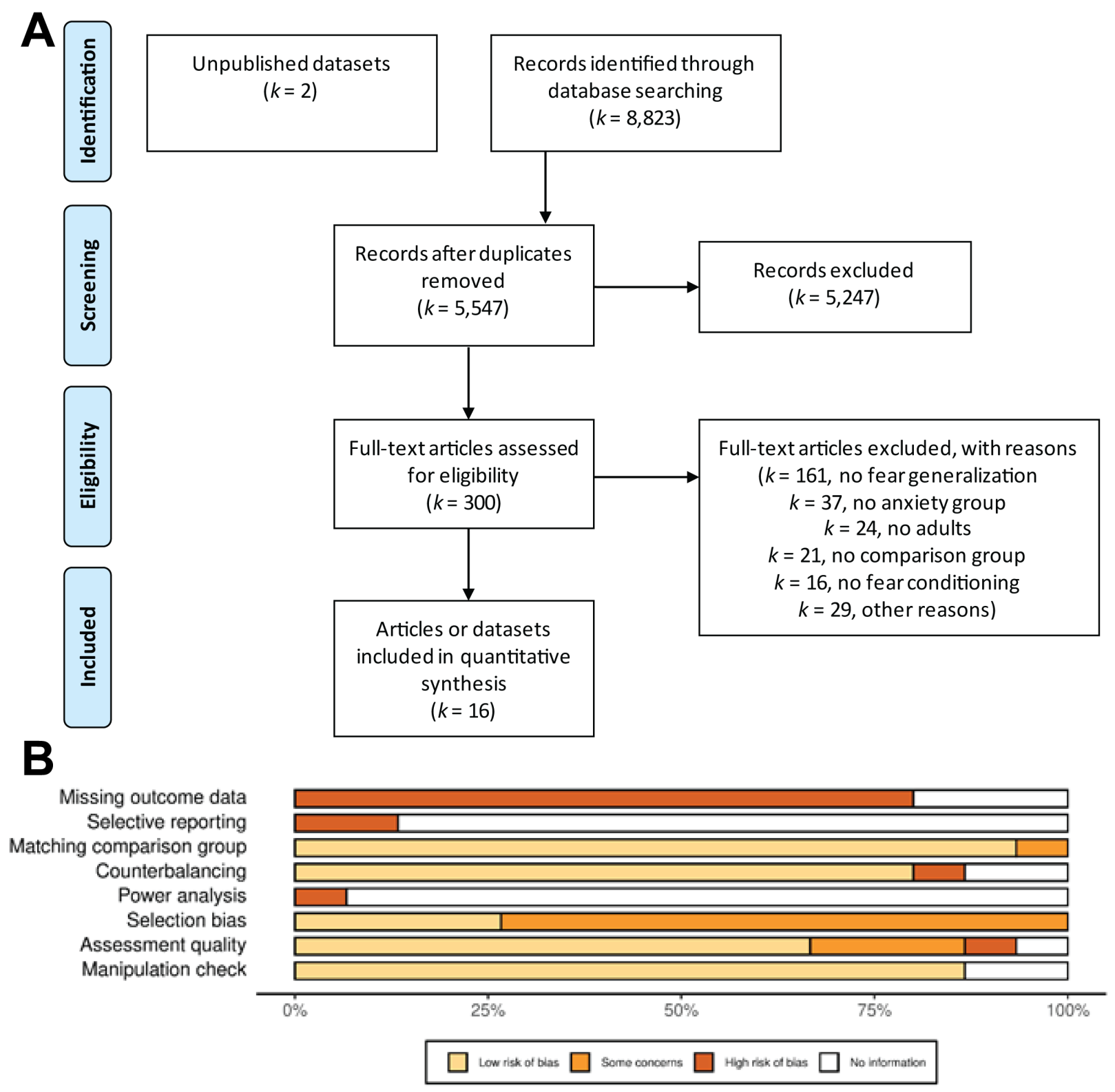

Note. PRISMA flow diagram (A) shows process of identification, screening, eligibility determination, and inclusion that lead to the final $N=16$ studies that were meta-analyzed. Panel B shows results from QA of included studies, which were evaluated by independent reviewers and assigned a rating of "low risk of bias", "some concerns", "high risk of bias", or "no information" for each criterion. Bars represent proportion of studies that obtained each rating on each criterion, with a great proportion of "low risk of bias" (pale yellow) indicative of higher overall study quality. Unpublished studies were not included in QA. PRISMA= Preferred Reporting Items for Systematic Reviews and Meta-Analyses; QA = quality assessment. 
Table 1. Characteristics of $N=16$ studies included in meta-analysis.

--For downloadable table, see:

https://mfr.osf.io/render?url=https://osf.io/7234h/?direct\%26mode=render\%26action=downloa $d \% 26$ mode $=$ render

\begin{abstract}
Abbreviations: $\mathrm{ACQ}=$ acquisition $; \mathrm{CAPS}=$ Clinician Administered PTSD Scale; $\mathrm{CS}=$ conditioned stimulus; $\mathrm{CS}+=$ conditioned threat cue; $\mathrm{CS}-=$ conditioned safety cue; $\mathrm{CR}=$ conditioned response; $\mathrm{CTX}=$ context; $\mathrm{fMRI}=$ functionalmagnetic resonance imaging; FPS = fear potentiated startle; GAD = genera lized anxiety disorder; GEN = generalization; $\mathrm{GS}$ = genera lization stimuli; $\mathrm{HAB}=$ habituation; $\mathrm{HR}=$ heart rate; $\mathrm{MINI}=$ Mini International Neuropsychiatric Inventory; $\mathrm{OCD}=$ obsessive-compulsive disorder; OCI-R $=$ Obsessive-Compulsive Disorder Inventory - Revised; $\mathrm{PA}=$ panic attacks; $\mathrm{PD}=$ panic disorder; $\mathrm{PRE}-\mathrm{ACQ}=$ pre-acquisition; $\mathrm{PTSD}=$ posttraumatic stress disorder; $\mathrm{SAD}=$ social anxiety disorder; $\mathrm{SCID}=$ Structured Clinical Interview for DSM; SCL = skin conductance level; $\mathrm{SCR}$ = skin conductance response; $\mathrm{SP}$ = specific phobia; SPIN - Social Phobia Inventory; US = unconditioned stimulus.
\end{abstract}




\section{Meta-Analyses of Full Data and Quadratic Interaction Effects}

\section{Primary Models}

Meta-analysis of the full data $(n=16, k=35)$ yielded a small effect size in favor of anxietyrelated disorder groups demonstrating heightened fear generalization compared with comparison participants, $g=.24,95 \% \mathrm{CI}_{\mathrm{c}-\mathrm{r}}[0.1,0.37], t(15)=3.84, p_{\mathrm{c}-\mathrm{r}}=.001$ (see Figure 3 ). Heterogeneity was nonsignificant, $\mathrm{I}^{2}=25.45 \%, Q(34)=47.39, p=0.063$. The quadratic interaction model $(n=9, k=15)$ yielded a larger effect size in the same direction as the full data model, $g=.3,95 \% \mathrm{CI}_{\mathrm{c}-\mathrm{r}}[0.02,0.58]$, $t(14)=2.31, p_{\mathrm{c}-\mathrm{r}}=.036$ (see Figure 3 ). Heterogeneity for this model was significant, $\mathrm{I}^{2}=58.45 \%$, $Q(14)=28.15, p=0.013$. Exclusion of the $\mathrm{n}=2$ unpublished studies resulted in larger meta-analytic effect sizes in both models but did not change direction or significance (see Supplementary Materials). 
Figure 3. Meta-analytic results by anxiety-related disorder diagnosis.

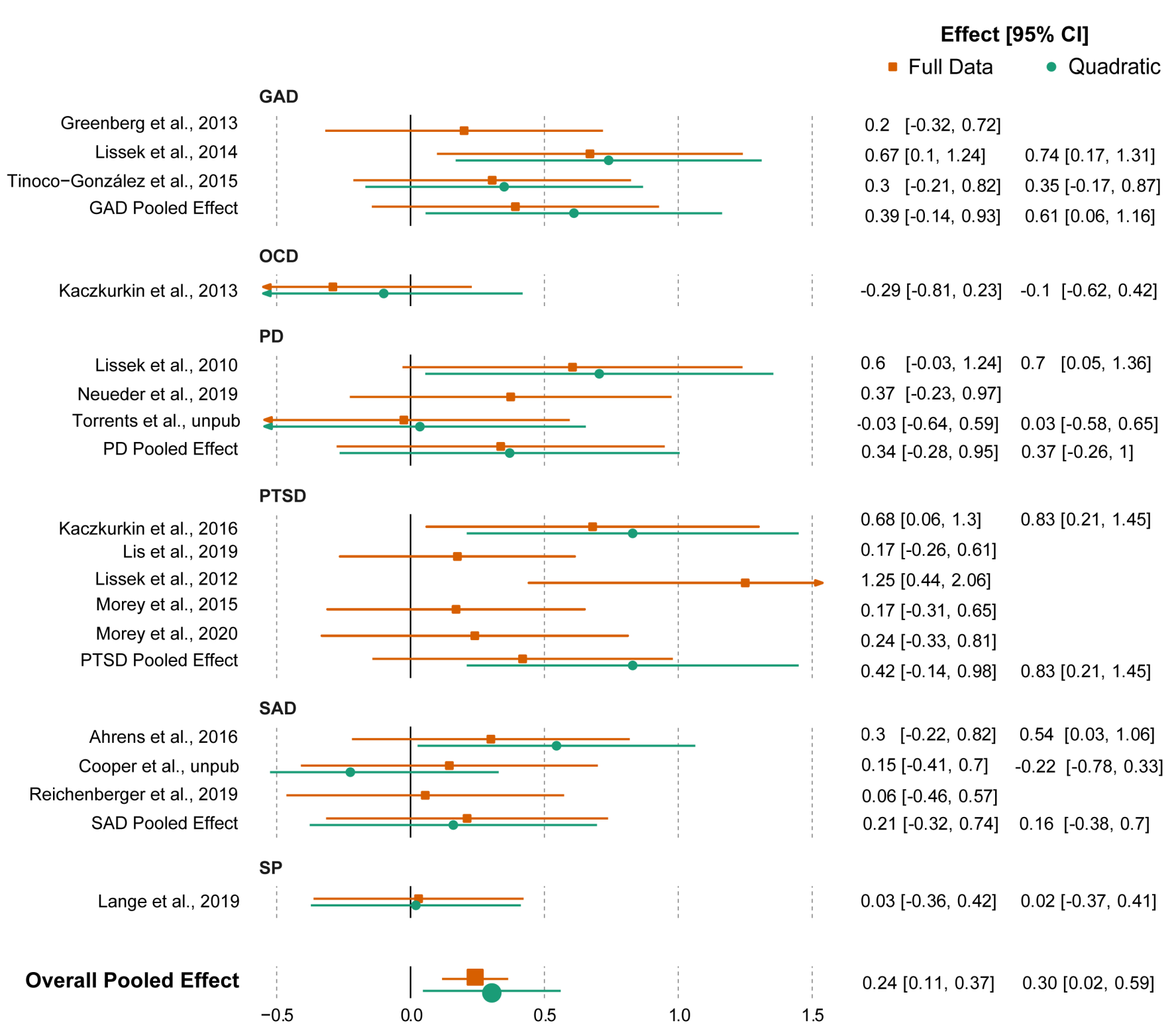

Notes. Plotted points represent effect sizes and $95 \%$ confidence intervals derived from random-effects meta-analytic models. Pooled effects are the avera ge effect size for each disorder; summary pooled effect represents a vera ge effect size across all disorders. $\mathrm{GAD}=$ genera lized anxiety disorder; $\mathrm{OCD}=$ obsessive-compulsive disorder; $\mathrm{PD}=$ panic disorder; $\mathrm{SAD}=$ social anxiety disorder; $\mathrm{SP}=$ specific phobia . 


\section{Moderation and Meta-Regressions}

All tested categorical moderators were non-significant (see Table 1). This included a lack of moderation by anxiety diagnosis, although some diagnoses had significant effect sizes when tested against zero (PTSD, SAD, GAD; all $\left.p_{f d r} \leq .048\right)$ in the full data model. Similarly, clinical status (threshold vs. sub-threshold) was not a significant moderator, but only studies with threshold samples were significant when the effect size was tested against zero (threshold: $p_{f d r}<.001$; subthreshold: $p_{f d r}=.765$ ). All continuous variables were also non-significant (all $p_{f d r}>.24$; see Table ST5)

\section{Publication Bias and $Q A$}

For both models, Rosenthal's fail-safe $\mathrm{N}$ and $p$-curve analyses indicated no risk of publication bias (see Supplementary Materials). Funnel-plots indicated significant asymmetry in both models driven by positive outliers (see Figures S5-S6). Removal of outliers in each analysis yielded smaller but still significant effect sizes (full-model: $g=.21$; quadratic model: $g=.26$ ). Relatedly, see Figures S7-S8 for sensitivity (“leave-one-out”) plots for all models.

\section{Discussion}

Improved understanding of the relationship between heightened fear generalization and anxiety-related disorders is an important step in the process of translating laboratory findings to clinical practice. Our primary hypothesis was supported: when meta-analyzing data from all 16 available studies, a small positive effect size was found in favor of heightened generalization in the anxiety-related disorder groups relative to comparison participants. This effect size increased in the meta-analysis that only tested quadratic effects, indicating that less curvilinear generalization gradients (i.e., shallower declines from CS+ to CS-, consistent with increased responding to GSs) differentiate anxiety-related disorder groups from comparison participants and might represent a 
more sensitive pathogenic marker of these pathologies. Heterogeneity ranged from negligible to moderate. Cross-study variations in conditioning parameters did not significantly moderate metaanalytic effect sizes. Similarly, within-study variation in dependent variables (physiological vs. subjective ratings) was not a significant moderator. Both results align with prior fear conditioning and generalization meta-analyses that did not find study design parameters significantly affected results (Duits et al., 2015; Lissek et al., 2005; Sep et al., 2019). Significant heterogeneity was, however, found in the quadratic model, which is possibly due to the smaller number of effect sizes analyzed relative to the full-data analysis and other fear conditioning meta-analyses. Overall, publication bias was largely acceptable across multiple indices. An exception was in our funnel plots, as extreme positive effect sizes were identified. That said, outlier removal did not significantly change results.

A key strength of the current effort is the breadth of designs and disorders we analyzed. In line with hypotheses and theory regarding the transdiagnostic import of fear generalization, anxiety-related disorder diagnosis did not moderate the observed meta-analytic effects. However, this does not mean that all diagnoses exhibited the same effect size, as can be seen in Table X. When comparing each diagnosis effect size to zero (i.e., a null effect) in isolation from other disorders, GAD and PTSD emerged as the two statistically strongest effect sizes (although caution in interpretation is needed due to the non-significant overall moderator). This is not unexpected, as etiological accounts of both disorders highlight fear generalization to a wide range of stimuli and contexts as central pathological factors (Zinbarg et al., 2022). Smaller effect sizes in other disorders are undoubtedly a function of smaller sample sizes (particularly for OCD and SP), but also potentially reflects that fear generalization functions more peripherally or is limited to fewer 
stimuli in these disorders, therefore limiting the capacity for laboratory tests to detect stronger effects.

The clinical status of the anxiety-related disorder group was also not a significant moderator. However, we found limited statistical evidence (effect sizes tested in isolation against zero) for stronger overall effect sizes for studies of threshold clinical groups compared to those that tested subclinical groups, but this again must be interpreted with caution due to the lack of an overall moderation effect. A recent meta-analysis of dimensional anxiety traits and fear generalization that explicitly excluded threshold or sub-threshold anxiety-related disorder data found a significant small and positive correlational effect size ( $r=.19)$ (Sep et al., 2019). Overall, meta-analytic evidence suggests that fear generalization varies as a function of anxiety symptom or trait severity and is not circumscribed to specific disorders. Although requiring further empirical work to verify, this pattern implies that heightened fear generalization is a dimensional phenotype that more closely relates to degree, as opposed to type, of anxiety pathology (Marin et al., 2020). Future studies might consider using assessments that measure a range of internalizing symptom dimensions (D. Watson et al., 2012) located within empirical psychopathology structures (e.g., the Hierarchical Taxonomy of Psychopathology, for recent review on internalizing in this model see D. Watson et al., 2022) to more precisely investigate the relation between fear generalization and cross-diagnostic and dimensional anxiety.

An important conclusion born from the current effort is the value of behavioral fear generalization paradigms using self-report or physiological measures for identifying abnormalities in threat reactivity associated with anxiety-related disorders. The analyzed data suggests these paradigms reliably provide a clinically-relevant behavioral marker that distinguishes between anxiety-related disorder patients and comparison participants. Further, researchers can expect a 
group difference effect size that is similar to or larger than those generated by differential conditioning paradigms. For example, the small effect size of $g=.24$ found for the full data analysis is larger than prior meta-analytic effect sizes of differential fear conditioning (CS+ vs. CS-, $|d|=.15)$ and slightly smaller than the effect size for CS- acquisition ( $d=.29$ ) (Duits et al., 2015). Promisingly, the quadratic effect analysis yielded one of the largest anxiety-related disorder metaanalytic effect sizes for a conditioning index in the literature to date, and the largest for an index that accounts for multiple stimuli. The overall largest is the effect size for CS + extinction $(d=.35)$, but this is a single cue index that does not consider other stimuli (e.g., CS-). Taken together, current results and the clinical relevance of fear generalization paradigms lend themselves well to psychiatric investigations, such as those measuring neuropathophysiological markers to use as predictors of the development of symptoms and of treatment effects.

\section{Fear Generalization in the Clinically Anxious Brain}

Improved understanding of the neural circuits that underlie fear generalization in clinical populations is crucial to inform precise models of neuropathophysiology that can then guide innovative therapies. The current meta-analysis was limited to behavioral and psychophysiological data; however, functional magnetic resonance imaging (fMRI) data were collected in five studies testing a limited number of anxiety-related disorders (Greenberg et al., 2013; Kaczkurkin et al., 2017; Lange, Goossens, Bakker, Michielse, Marcelis, et al., 2019; Morey et al., 2015, 2020) (see Table 2). Due to the small number of studies with relevant data, and an expected small-to-moderate effect and the associated imaging meta-analysis power concerns (Müller et al., 2018), we did not perform a quantitative analysis of fMRI data. Instead, we provide a brief qualitative assessment. 
Table 2. Overview of key findings from fMRI studies of fear generalization in anxiety-related disorders.

\begin{tabular}{|c|c|c|c|c|c|c|c|c|c|}
\hline $\begin{array}{l}\text { Generalization Task } \\
\text { Perceptual }\end{array}$ & $\underline{\mathrm{Dx}}$ & $\underline{\mathrm{ACC}}$ & Amygdala & $\underline{\text { Striatum }}$ & $\underline{\mathrm{dmPFC} / \mathrm{SMA}}$ & Hippocampus & $\underline{\text { Insula }}$ & $\underline{\text { Thalamus }}$ & vmPFC \\
\hline Greenberg et al., 2013 & GAD & $\uparrow$ & - & $\uparrow$ & $\uparrow$ & - & $\uparrow$ & - & $\downarrow$ \\
\hline Kaczkurkin et al., 2016 & PTSD & - & - & $\uparrow$ & $\uparrow$ & $\downarrow$ & $\uparrow$ & $\uparrow$ & - \\
\hline Lange et al., 2019 & SP & - & - & - & - & - & - & - & $\downarrow$ \\
\hline Morey et al., 2015 & PTSD & - & $\uparrow$ & - & - & - & $\downarrow$ & $\uparrow$ & $\downarrow^{1}$ \\
\hline \multicolumn{10}{|l|}{ Conceptual } \\
\hline Morey et al., 2020 & PTSD & $\uparrow$ & $\uparrow$ & $\uparrow$ & - & - & $\uparrow$ & - & $\uparrow$ \\
\hline
\end{tabular}

Notes: Regions of interest to be included here were selected based on a meta-analysis of seven fMRI fear generalization studies in psychiatrically healthy samples identified significant nodes within cingulo-opercular (salience) and frontoparietal (central executive, default mode) networks that compete to express or impede fearful behavior when confronted with a generalization stimulus (Webler et al., 2021). Primary regions of interest in these circuits include those related to the evaluation and awareness of threat (e.g., amygdala, insula, ACC dorsal medial prefrontal cortex [dmPFC]) and associated fear-related actions (e.g., caudate, supplementary motor area [SMA]). Also included are those related to safety processing (e.g., vmPFC) regions that work to discern between threat and safety (e.g., hippocampus) and those that facilitate activity throughout the involved circuitry (e.g., thalamus).

Hyperactivity in the anxiety group relative to the control group is indicated with an upward arrow; hypoactivity with a downward arrow. Here, ACC refers to all reported subdivisions, and striatum includes caudate and putamen. $\mathrm{ACC}=$ anterior cingulate cortex; $\mathrm{dmPFC}=$ dorsomedial prefrontal cortex; $\mathrm{Dx}=$ anxiety diagnosis; $\mathrm{GAD}=$ generalized anxiety disorder; $\mathrm{PTSD}$ $=$ posttraumatic stress disorder; $\mathrm{SMA}=$ supplementary motor area; $\mathrm{SP}=$ specific phobia; $\mathrm{vmPFC}=$ ventromedial prefrontal cortex.

${ }^{1}$ Decreased functional connectivity with amygdala in PTSD > control; no univariate differences found. 
The reviewed fMRI data provide evidence for some, but not all, of the cingulo-opercular and frontoparietal regions implicated in studies of fear generalization in psychiatrically healthy people (Webler et al., 2021) as significantly related to an anxiety-related disorder during a fear generalization task (see Figure 4). The most consistent cross-study findings were hypoactive ventromedial prefrontal cortex (vmPFC), hyperactive striatal regions (e.g., caudate, putamen), and primarily hyperactivation in the insula to be related to anxiety-related disorders. Due to their prominence in animal work and theoretical accounts of generalization, the lack of consistent amygdala and hippocampus findings in the reviewed studies is notable. Higher-resolution fMRI studies that can reliably detect activity in functional subregions with more specific relations to generalization processes (e.g., lateral central amygdala, hippocampal subfields) (Huggins et al., 2021) are needed. However, broad conclusions regarding all fMRI findings are preliminary, especially considering the small number of identified studies that cover only some disorders.

In terms of the current behavioral meta-analysis, the fMRI findings are generally in line with the finding of heightened fear generalization in anxiety-related disorders, as neural regions that were related to fear generalization, such as the insula and striatum, are associated with behavioral measures of conditioned responding (Schiller \& Delgado, 2010). The fMRI findings also highlight a notable weakness of the analyzed behavioral data: psychophysiological and rating measures cannot disentangle excitatory and inhibitory contributions to fear generalization, whereas fMRI is well-suited to this goal. In their meta-analysis, Webler et al. (2021) identified regions associated with increased (excitatory) and decreased (inhibitory) generalization gradients. The behavioral fear generalization effect size detected in the current analyses might change in magnitude or be moderated by clinical parameters if these two types of generalization gradients 
were examined separately. A future meta-analysis of fMRI data, once sufficient studies are available, would be an important next step for this area.

Figure 4. Visualization of cross-study neural regions significantly differentiating anxiety-related disorder from comparison participants.

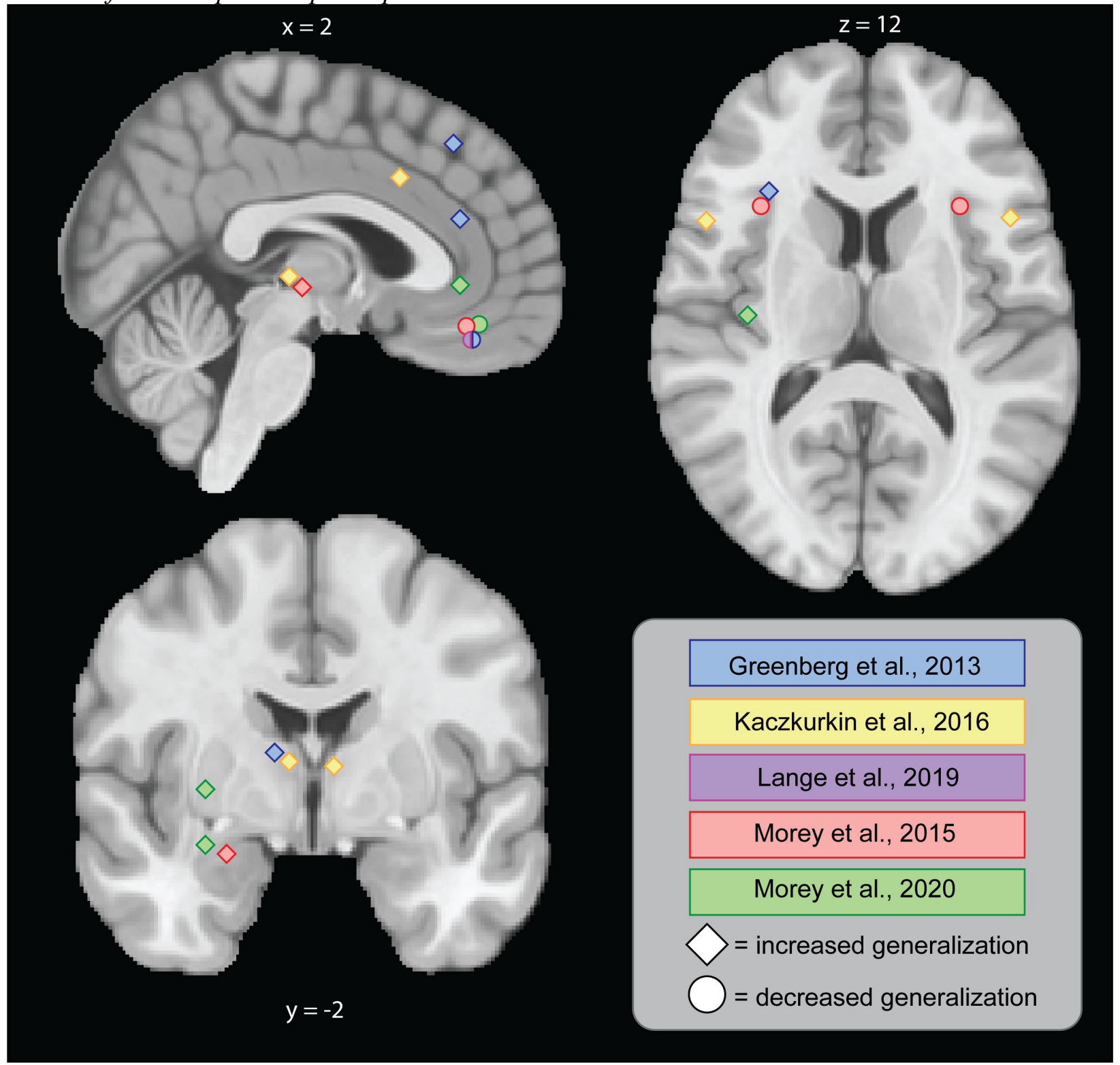

Notes. All points are centered on peak activation coordinates (MNI space) reported in reviewed studies; the size of each point is fixed and does not correspond to an empirical value. Regions rela ted to increased genera lization (i.e., genera lization values decrease para metrically from $\mathrm{CS}+$ ) are demarcated by diamonds; regions related to decreased generalization (i.e., genera lization values increase parametrically from $\mathrm{CS}+$ ) are demarcated by circles. Halved points comprised of two different colors were included when two studies reported the same MNI coordinates for a given region. 


\section{Current and Future Challenges}

\section{Limitations}

Fear generalization research in clinical populations is a growing field, and the limitations of the present effort reflect this. First, the available number of studies complicates fine-grained examination of subtle mechanisms and variations of fear generalization in anxiety-related disorders. Our primary analysis of standard interaction effects can only speak to the overall magnitude of generalization differences between anxiety and comparison groups, not to differences in the shape of generalization gradients. Crucially, this is a coarse metric that does not statistically differentiate between GSs and CS+/CS-, and therefore, non-generalization processes (e.g., sensitization) might be present to an extent. More linear or less curvilinear (i.e., GSs more similar to $\mathrm{CS}+$ elicit responses closer in magnitude to the $\mathrm{CS}+$ ) generalization gradients are proposed as a pathogenic marker of those with anxiety-related disorders (Lissek, 2012). Our analysis of quadratic effects in the subset of studies with relevant data supports this assertion, but was not optimized to detect subtle individual differences in gradient shape related to one or more GSs. Further, the meaning and quantification of generalization gradients is an ongoing topic of empirical discussion (Lee et al., 2020; Vanbrabant et al., 2015). Thus, we cannot state that anxietyrelated disorders are clearly defined by a particular gradient shape.

Second, for some disorders (e.g., OCD, SP), there were not enough studies to make sound conclusions regarding their relation to fear generalization at this time; thus, caution is needed in interpreting effect sizes from specific disorders. We also note that caution is generally needed when interpreting moderation effects in the meta-analytic framework, particularly with the relatively small number of studies in the current effort (Borenstein \& Higgins, 2013). There is also the issue of potentially impactful moderators that were not assessed in the original studies and 
therefore were not tested (e.g., childhood maltreatment, Lange, Goossens, Bakker, Michielse, van Winkel, et al., 2019; Teicher et al., 2021). Finally, we were not able to fully test the impact of specific comorbid disorders (e.g., depressive disorders) and psychoactive medications in our metaregressions due to small sample size or imbalances in study protocols.

\section{Open Science Practices and Study Quality}

Our QA found some trends that merit discussion, particularly in the context of increasing open science practices (Nosek et al., 2015). On the one hand, most articles reported having matched their comparison groups, having counterbalanced their stimuli, and having used validated assessment instruments - which all contribute to quality and reliability. On the other hand, however, some risks of bias should be noted. First, no studies preregistered their hypotheses regarding fear generalization. Although a recent development, this open science practice is crucial for improving the trustworthiness of published findings (Bell, 2017). Second, no studies reported an a priori power analysis, and, given their relatively small sample sizes, they might be statistically underpowered. Compounding this issue is that the reliability of meta-analyses might be reduced when including many studies with small sample sizes, as they often have stronger heterogeneity or chance findings (i.e., small-study effect) (Nuijten et al., 2020). Third, reasons for exclusions and for missing datadiffered across each study or were not reported. More transparency on treating excluded or missing data might increase the integrity of findings and across-study comparability.

\section{Future Directions}

In addition to the future directions and challenges outlined above, it is important to emphasize a key opportunity for future work. The current meta-analysis does not explain the how of heightened fear generalization in anxiety-related disorders, and this remains an important next step. All analyzed studies were cross-sectional, and behavioral measures do not allow us to 
disentangle specific mechanisms of the fear generalization process (as can be done with neuroimaging). This also means we cannot yet determine if heightened fear generalization is one of, or a combination of, the following: a predisposing risk factor for anxiety-related pathology, a pathogenic mechanism, or a manifest symptom of the pathology. Further, we do not know if generalization deficits are isolated to threat and fear or if there are non-affective dysfunctions that serve to predispose people towards heightened generalization (e.g., perceptual discrimination difficulties; Struyf et al., 2017). Additional studies using prospective longitudinal designs, testing other forms of generalization and related processes, and linking fear generalization task data to clinical data are needed to comprehensively address this important issue. In line with previously addressed limitations, we also recommend that these future studies, when feasible, use dimensional assessments and neuroimaging (e.g., fMRI) to facilitate precise measurement of the underlying components of fear generalization and their relations to fine-grained markers of anxiety psychopathology.

\section{Conclusion}

Overall, it is clear there are many future avenues generalization research can take, and the current meta-analytic results suggest that conditioning researchers might strongly consider using a generalization design instead of simple differential conditioning. We contend that fear generalization has notable empirical and conceptual advantages over differential acquisition and extinction paradigms and is a viable model for clinical translational research on emotional memory and learning in anxiety-related disorders. We anticipate that acceleration of generalization research will facilitate innovation and advancements in conceptualization and treatment of clinical anxiety and related conditions 


\section{Acknowledgments}

We thank Sophie Bergmeijer, Mona Laskar, Annemijn Hoekman, Michelle Gersmann, and Layla Vergeer for their assistance in the article screening and selection process. We also thank Ryan Webler, Nicole Keller, and Gus Hennings for helpful discussions regarding fMRI results and figures, and anonymous reviewers for their comments that have substantially improved this manuscript

This work was supported by the National Institutes of Health (F32 MH129136, awarded to SEC; R01 MH122387, awarded to JED) and National Science Foundation (CAREER Award 1844792, awarded to JED). 


\section{Financial Disclosures}

Dr. Nemeroff has received research support from NIH; he has served as a consultant for ANeuroTech (division of Anima BV), Signant Health, Magstim, Inc., Navitor Pharmaceuticals, Inc., Intra-Cellular Therapies, Inc., EMA Wellness, Acadia Pharmaceuticals, Sage, BioXcel Therapeutics, Silo Pharma, XW Pharma, Neuritek, Engrail Therapeutics, Corcept Therapeutics Pharmaceuticals Company, SK Life Science, Alfasigma, Pasithea Therapeutic Corp., EcoR1; he has served on scientific advisory boards for the ANeuroTech (division of Anima BV), Brain and Behavior Research Foundation (BBRF), Anxiety and Depression Association of America (ADAA), Skyland Trail, Signant Health, Laureate Institute for Brain Research (LIBR), Inc., Magnolia CNS, Heading Health, TRUUST Neuroimaging, Pasithea Therapeutic Corp.; he is a stockholder in Xhale, Seattle Genetics, Antares, BI Gen Holdings, Inc., Corcept Therapeutics Pharmaceuticals Company, EMA Wellness, TRUUST Neuroimaging; he serves on the board of directors for Gratitude America, ADAA, Xhale Smart, Inc., Lucy Scientific Discovery, Inc; and he holds patents on a method and devices for transdermal delivery of lithium (patent 6,375,990B1) and a method of assessing antidepressant drug therapy via transport inhibition of monoamine neurotransmitters by ex vivo assay (patent 7,148,027B2).

The other authors report no financial relationships with commercial interests. 


\section{References}

Ahrens, L. M., Pauli, P., Reif, A., Mühlberger, A., Langs, G., Aald erink, T., \& Wieser, M. J. (2016). Fear conditioning and stimulus generalization in patients with social anxiety disorder. Journal of Anxiety Disorders, 44, 36-46.

https://doi.org/10.1016/j.janxdis.2016.10.003

American Psychiatric Association. (2013). Diagnostic and statistical manual of mental disorders (Fifth Edition). American Psychiatric Association. http://psychiatryonline.org/doi/book/10.1176/appi.books.9780890425596

Arch, J. J., \& Abramowitz, J. S. (2015). Exposure therapy for obsessive-compulsive disorder: An optimizing inhibitory learning approach. Journal of Obsessive-Compulsive and Related Disorders, 6, 174-182. https://doi.org/10.1016/j.jocrd.2014.12.002

Asmundson, G. J. G. (2019). A rose by any other name...How should we refer to the collective of conditions characterized by clinically significant anxiety? Journal of Anxiety Disorders, 68, 102143. https://doi.org/10.1016/j.janxdis.2019.102143

Bandelow, B., \& Michaelis, S. (2015). Epidemiology of anxiety disorders in the 21 st century. Dialogues in Clinical Neuroscience, 17(3), 327-335.

Baxter, A. J., Scott, K. M., Vos, T., \& Whiteford, H. A. (2013). Global prevalence of anxiety disorders: A systematic review and meta-regression. Psychological Medicine, 43(5), 897910. https://doi.org/10.1017/S003329171200147X

Beckers, T., Krypotos, A.-M., Boddez, Y., Effting, M., \& Kindt, M. (2013). What's wrong with fear conditioning? Biological Psychology, 92(1), 90-96. https://doi.org/10.1016/j.biopsycho.2011.12.015 
Bell, V. (2017). Open science in mental health research. The Lancet Psychiatry, 4(7), 525-526. https://d oi.org/10.1016/S2215-0366(17)30244-4

Besnard, A., \& Sahay, A. (2016). Adult Hippocampal Neurogenesis, Fear Generalization, and Stress. Neuropsychopharmacology, 41(1), 24-44. https://doi.org/10.1038/npp.2015.167

Borenstein, M., \& Higgins, J. P. T. (2013). Meta-Analysis and Subgroups. Prevention Science, 14(2), 134-143. https://doi.org/10.1007/s11121-013-0377-7

Cafri, G., Kromrey, J. D., \& Brannick, M. T. (2010). A Meta-Meta-Analysis: Empirical Review of Statistical Power, Type I Error Rates, Effect Sizes, and Model Selection of MetaAnalyses Published in Psychology. Multivariate Behavioral Research, 45(2), 239-270. https://doi.org/10.1080/00273171003680187

Cameron, A. C., \& Miller, D. L. (2015). A Practitioner's Guide to Cluster-Robust Inference. Journal of Human Resources, 50(2), 317-372. https://doi.org/10.3368/jhr.50.2.317

Cooper, S. E., \& Dunsmoor, J. E. (2021). Fear conditioning and extinction in obsessivecompulsive disorder: A systematic review. Neuroscience \& Biobehavioral Reviews, 129, 75-94. https://doi.org/10.1016/j.neubiorev.2021.07.026

Craske, M. G., Hermans, D., \& Vervliet, B. (2018). State-of-the-art and future directions for extinction as a translational model for fear and anxiety. Philosophical Transactions of the Royal Society B: Biological Sciences, 373(1742), 20170025. https://doi.org/10.1098/rstb.2017.0025

Duits, P., Cath, D. C., Lissek, S., Hox, J. J., Hamm, A. O., Engelhard, I. M., van den Hout, M. A., \& Baas, J. M. P. (2015). Updated meta-analysis of classical fear conditioning in the anxiety disorders. Depression and Anxiety, 32(4), 239-253.

https://doi.org/10.1002/da.22353 
Dunsmoor, J. E., Niv, Y., Daw, N., \& Phelps, E. A. (2015). Rethinking Extinction. Neuron, 88(1), 47-63. https://doi.org/10.1016/j.neuron.2015.09.028

Dunsmoor, J. E., \& Paz, R. (2015). Fear Generalization and Anxiety: Behavioral and Neural Mechanisms. Biological Psychiatry, 78(5), 336-343. https://doi.org/10.1016/j.biopsych.2015.04.010

Dymond, S., Dunsmoor, J. E., Vervliet, B., Roche, B., \& Hermans, D. (2015). Fear Generalization in Humans: Systematic Review and Implications for Anxiety Disorder Research. Behavior Therapy, 46(5), 561-582. https://doi.org/10.1016/j.beth.2014.10.001

Foa, E. B., \& McLean, C. P. (2016). The Efficacy of Exposure Therapy for Anxiety-Related Disorders and Its Underlying Mechanisms: The Case of OCD and PTSD. Annual Review of Clinical Psychology, 12(1), 1-28. https://d oi.org/10.1146/annurev-clinpsy-021815093533

Greenberg, T., Carlson, J. M., Cha, J., Hajcak, G., \& Mujica-Parodi, L. R. (2013). Ventromedial Prefrontal Cortex Reactivity Is Altered in Generalized Anxiety Disorder During Fear Generalization. Depression and Anxiety, 30(3), 242-250. https://doi.org/10.1002/da.22016

Grupe, D. W., \& Nitschke, J. B. (2013). Uncertainty and anticipation in anxiety: An integrated neurobiological and psychological perspective. Nature Reviews Neuroscience, 14(7), 488-501. https://doi.org/10.1038/nrn3524

Higgins, J. P. T., Altman, D. G., Gøtzsche, P. C., Jüni, P., Moher, D., Oxman, A. D., Savović, J., Schulz, K. F., Weeks, L., \& Sterne, J. A. C. (2011). The Cochrane Collaboration's tool for assessing risk of bias in randomised trials. $B M J, 343$. https://doi.org/10.1136/bmj.d5928 
Holmes, E. A., Ghaderi, A., Harmer, C. J., Ramchandani, P. G., Cuijpers, P., Morrison, A. P., Roiser, J. P., Bockting, C. L. H., O’Connor, R. C., Shafran, R., Moulds, M. L., \& Craske, M. G. (2018). The Lancet Psychiatry Commission on psychological treatments research in tomorrow's science. The Lancet Psychiatry, 5(3), 237-286.

https://doi.org/10.1016/S2215-0366(17)30513-8

Huggins, A. A., Weis, C. N., Parisi, E. A., Bennett, K. P., Miskovic, V., \& Larson, C. L. (2021). Neural Substrates of Human Fear Generalization: A 7T-fMRI Investigation. NeuroImage, 118308. https://doi.org/10.1016/j.neuroimage.2021.118308

Kaczkurkin, A. N., Burton, P. C., Chazin, S. M., Manbeck, A. B., Espensen-Sturges, T., Cooper, S. E., Sponheim, S. R., \& Lissek, S. (2017). Neural substrates of overgeneralized conditioned fear in PTSD. American Journal of Psychiatry, 174(2), 125-134. https://doi.org/10.1176/appi.ajp.2016.15121549

Kaczkurkin, A. N., \& Lissek, S. (2013). Generalization of conditioned fear and obsessivecompulsive traits. Journal of Psychology \& Psychotherapy, 7, 3.

Lakens, D. (2013). Calculating and reporting effect sizes to facilitate cumulative science: A practical primer for t-tests and ANOVAs. Frontiers in Psychology, 4. https://d oi.org/10.3389/fpsyg.2013.00863

Lange, I., Goossens, L., Bakker, J., Michielse, S., Marcelis, M., Wichers, M., van Os, J., van Amelsvoort, T., \& Schruers, K. (2019). Functional neuroimaging of associative learning and generalization in specific phobia. Progress in Neuro-Psychopharmacology and Biological Psychiatry, 89, 275-285. https://doi.org/10.1016/j.pnpbp.2018.09.008

Lange, I., Goossens, L., Bakker, J., Michielse, S., van Winkel, R., Lissek, S., Leibold, N., Marcelis, M., Wichers, M., van Os, J., van Amelsvoort, T., \& Schruers, K. (2019). 
Neurobehavioural mechanisms of threat generalization moderate the link between childhood maltreatment and psychopathology in emerging adulthood. Journal of Psychiatry \& Neuroscience : JPN, 44(3), 185-194. https://doi.org/10.1503/jpn.180053

LeDoux, J. E. (1994). Emotion, Memory and the Brain. Scientific American, 270(6), 50-57.

Lee, J. C., Mills, L., Hayes, B. K., \& Livesey, E. J. (2020). Modelling generalisation gradients as augmented Gaussian functions. Quarterly Journal of Experimental Psychology, 1747021820949470. https://doi.org/10.1177/1747021820949470

Lis, S., Thome, J., Kleindienst, N., Mueller-Engelmann, M., Steil, R., Priebe, K., Schmahl, C., Hermans, D., \& Bohus, M. (2019). Generalization of fear in post-traumatic stress disorder. Psychophysiology, e13422.

Lissek, S. (2012). Toward an Account of Clinical Anxiety Predicated on Basic, Neurally Mapped Mechanisms of Pavlovian Fear-Learning: The Case for Conditioned Overgeneralization. Depression and Anxiety, 29(4), 257-263. https://doi.org/10.1002/da.21922

Lissek, S., \& Grillon, C. (2012). Learning models of PTSD. The Oxford Handbook of Traumatic Stress Disorders. https://doi.org/10.1093/oxfordhb/9780195399066.013.0013

Lissek, S., Kaczkurkin, A. N., Rabin, S., Geraci, M., Pine, D. S., \& Grillon, C. (2014). Generalized anxiety disorder is associated with overgeneralization of classically conditioned fear. Biological Psychiatry, 75(11), 909-915. https://doi.org/10.1016/j.biopsych.2013.07.025

Lissek, S., Pine, D. S., \& Grillon, C. (2006). The strong situation: A potential impediment to studying the psychobiology and pharmacology of anxiety disorders. Biological Psychology, 72(3), 265-270. 
Lissek, S., Powers, A. S., McClure, E. B., Phelps, E. A., Woldehawariat, G., Grillon, C., \& Pine, D. S. (2005). Classical fear conditioning in the anxiety disorders: A meta-analysis. Behaviour Research and Therapy, 43(11), 1391-1424. https://doi.org/10.1016/j.brat.2004.10.007

Lissek, S., Rabin, S., Heller, R. E., Lukenbaugh, D., Geraci, M., Pine, D. S., \& Grillon, C. (2010). Overgeneralization of conditioned fear as a pathogenic marker of panic disorder. American Journal of Psychiatry, 167(1), 47-55. https://doi.org/10.1176/appi.ajp.2009.09030410

Lissek, S., \& van Meurs, B. (2014). Learning models of PTSD: Theoretical accounts and psychobiological evidence. International Journal of Psychophysiology. https://doi.org/10.1016/j.jpsycho.2014.11.006

Lopresto, D., Schipper, P., \& Homberg, J. R. (2016). Neural circuits and mechanisms involved in fear generalization: Implications for the pathophysiology and treatment of posttraumatic stress disorder. Neuroscience \& Biobehavioral Reviews, 60, 31-42. https://doi.org/10.1016/j.neubiorev.2015.10.009

Marin, M.-F., Hammoud, M. Z., Klumpp, H., Simon, N. M., \& Milad, M. R. (2020). Multimodal Categorical and Dimensional Approaches to Understanding Threat Conditioning and Its Extinction in Individuals With Anxiety Disorders. JAMA Psychiatry. https://doi.org/10.1001/jamapsychiatry.2019.4833

Milad, M. R., \& Quirk, G. J. (2012). Fear Extinction as a Model for Translational Neuroscience: Ten Years of Progress. Annual Review of Psychology, 63(1), 129-151. https://doi.org/10.1146/annurev.psych.121208.131631 
Morey, R. A., Dunsmoor, J. E., Haswell, C. C., Brown, V. M., Vora, A., Weiner, J., Stjepanovic, D., Wagner, H. R., Brancu, M., Marx, C. E., Naylor, J. C., Voorhees, E. V., Taber, K. H., Beckham, J. C., Calhoun, P. S., Fairbank, J. A., Szabo, S. T., \& LaBar, K. S. (2015). Fear learning circuitry is biased toward generalization of fear associations in posttraumatic stress disorder. Translational Psychiatry, 5(12), e700. https://doi.org/10.1038/tp.2015.196

Morey, R. A., Haswell, C. C., Stjepanović, D., Dunsmoor, J. E., LaBar, K. S., \& Mid-Atlantic MIRECC Workgroup,. (2020). Neural correlates of conceptual-level fear generalization in posttraumatic stress disorder. Neuropsychopharmacology, 45(8), 1380-1389. https://doi.org/10.1038/s41386-020-0661-8

Müller, V. I., Cieslik, E. C., Laird, A. R., Fox, P. T., Radua, J., Mataix-Cols, D., Tench, C. R., Yarkoni, T., Nichols, T. E., Turkeltaub, P. E., Wager, T. D., \& Eickhoff, S. B. (2018). Ten simple rules for neuroimaging meta-analysis. Neuroscience and Biobehavioral Reviews, 84, 151-161. https://doi.org/10.1016/j.neubiorev.2017.11.012

Neueder, D., Andreatta, M., \& Pauli, P. (2019). Contextual Fear Conditioning and Fear Generalization in Individuals With Panic Attacks. Frontiers in Behavioral Neuroscience, 13. https://doi.org/10.3389/fnbeh.2019.00152

Nosek, B. A., Alter, G., Banks, G. C., Borsboom, D., Bowman, S. D., Breckler, S. J., Buck, S., Chambers, C. D., Chin, G., Christensen, G., Contestabile, M., Dafoe, A., Eich, E., Freese, J., Glennerster, R., Goroff, D., Green, D. P., Hesse, B., Humphreys, M., ... Yarkoni, T. (2015). Promoting an open research culture. Science, 348(6242), 1422-1425. https://doi.org/10.1126/science.aab2374 
Nuijten, M. B., van Assen, M. A. L. M., Augusteijn, H. E. M., Crompvoets, E. A. V., \& Wicherts, J. M. (2020). Effect Sizes, Power, and Biases in Intelligence Research: A Meta-Meta-Analysis. Journal of Intelligence, 8(4), 36. https://doi.org/10.3390/jintelligence8040036

Pavlov, I. P. (1927). Conditioned reflexes. Courier Dover Publications.

Pittig, A., Treanor, M., LeBeau, R. T., \& Craske, M. G. (2018). The role of associative fear and avoidance learning in anxiety disorders: Gaps and directions for future research. Neuroscience \& Biobehavioral Reviews, 88, 117-140. https://doi.org/10.1016/j.neubiorev.2018.03.015

R Core Team. (2018). $R$ : A language and environment for statistical computing. R Foundation for Statistical Computing. https://www.R-project.org/

Reichenberger, J., Pfaller, M., Forster, D., Gerczuk, J., Shiban, Y., \& Mühlberger, A. (2019). Men Scare Me More: Gender Differences in Social Fear Conditioning in Virtual Reality. Frontiers in Psychology, 10. https://doi.org/10.3389/fpsyg.2019.01617

RoB 2: A revised Cochrane risk-of-bias tool for randomized trials $\mid$ Cochrane Bias. (n.d.). Retrieved February 23, 2022, from https://method s.cochrane.org/bias/resources/rob-2revised-cochrane-risk-bias-tool-randomized-trials

Schiller, D., \& Delgado, M. R. (2010). Overlapping neural systems mediating extinction, reversal and regulation of fear. Trends in Cognitive Sciences, 14(6), 268-276. https://doi.org/10.1016/j.tics.2010.04.002

Sep, M. S. C., Steenmeijer, A., \& Kennis, M. (2019). The relation between anxious personality traits and fear generalization in healthy subjects: A systematic review and meta-analysis. 
Neuroscience \& Biobehavioral Reviews, 107, 320-328.

https://doi.org/10.1016/j.neubiorev.2019.09.029

Struyf, D., Zaman, J., Hermans, D., \& Vervliet, B. (2017). Gradients of fear: How perception influences fear generalization. Behaviour Research and Therapy, 93, 116-122. https://doi.org/10.1016/j.brat.2017.04.001

Teicher, M. H., Gordon, J. B., \& Nemeroff, C. B. (2021). Recognizing the importance of childhood maltreatment as a critical factor in psychiatric diagnoses, treatment, research, prevention, and education. Molecular Psychiatry, 1-8. https://doi.org/10.1038/s41380$021-01367-9$

Tinoco-González, D., Fullana, M. A., Torrents-Rodas, D., Bonillo, A., Vervliet, B., Blasco, M. J., Farré, M., \& Torrubia, R. (2015). Conditioned Fear Acquisition and Generalization in Generalized Anxiety Disorder. Behavior Therapy, 46(5), 627-639. https://doi.org/10.1016/j.beth.2014.12.004

Vanbrabant, K., Boddez, Y., Verduyn, P., Mestdagh, M., Hermans, D., \& Raes, F. (2015). A new approach for modeling generalization gradients: A case for hierarchical models. Frontiers in Psychology, 6. https://doi.org/10.3389/fpsyg.2015.00652

Vervliet, B., \& Boddez, Y. (2020). Memories of 100 years of human fear conditioning research and expectations for its future. Behaviour Research and Therapy, 135, 103732. https://doi.org/10.1016/j.brat.2020.103732

Watson, D., Levin-Aspenson, H. F., Waszczuk, M. A., Conway, C. C., Dalgleish, T., Dretsch, M. N., Eaton, N. R., Forbes, M. K., Forbush, K. T., Hobbs, K. A., Michelini, G., Nelson, B. D., Sellbom, M., Slade, T., South, S. C., Sunderland, M., Waldman, I., Witthöft, M., Wright, A. G. C., ... Workgroup, H. U. (2022). Validity and utility of Hierarchical 
Taxonomy of Psychopathology (HiTOP): III. Emotional dysfunction superspectrum. World Psychiatry, 21(1), 26-54. https://doi.org/10.1002/wps.20943

Watson, D., O’Hara, M. W., Naragon-Gainey, K., Koffel, E., Chmielewski, M., Kotov, R., Stasik, S. M., \& Ruggero, C. J. (2012). Development and Validation of New Anxiety and Bipolar Symptom Scales for an Expanded Version of the IDAS (the IDAS-II). Assessment, 19(4), 399-420. https://doi.org/10.1177/1073191112449857

Watson, J. B., \& Rayner, R. (1920). Conditioned emotional reactions. Journal of Experimental Psychology, 3(1), 1-14. https://doi.org/10.1037/h0069608

Webler, R. D., Berg, H., Fhong, K., Tuominen, L., Holt, D. J., Morey, R. A., Lange, I., Burton, P. C., Fullana, M. A., Radua, J., \& Lissek, S. (2021). The neurobiology of human fear generalization: Meta-analysis and working neural model. Neuroscience \& Biobehavioral Reviews, 128, 421-436. https://doi.org/10.1016/j.neubiorev.2021.06.035

Zinbarg, R. E., Williams, A. L., \& Mineka, S. (2022). A Current Learning Theory Approach to the Etiology and Course of Anxiety and Related Disorders. Annual Review of Clinical Psychology. https://doi.org/10.1146/annurev-clinpsy-072220-021010

Zuj, D. V., Palmer, M. A., Lommen, M. J. J., \& Felmingham, K. L. (2016). The centrality of fear extinction in linking risk factors to PTSD: A narrative review. Neuroscience \& Biobehavioral Reviews, 69, 15-35. https://doi.org/10.1016/j.neubiorev.2016.07.014 